\title{
Haptic System for Determining the Young Modulus of Materials
}

\author{
http://dx.doi.org/10.3991/ijoe.v9iS8.3394 \\ Maria Teresa Restivo, António M. Lopes, Linda Padilla, Pedro Chaves and Teresa Duarte \\ University of Porto, Porto, Portugal
}

\begin{abstract}
This work describes the HapticBender: a haptic virtual experiment for determining the Young Modulus of materials. The Young Modulus is related to the stiffness of a material and is one of the most important properties of materials in the area of Materials Engineering and Technological Processes. The experiment is an evolution of an existing system where new features have been integrated. The HapticBender has been assessed trough the analysis of questionnaires answered by students sample that performed experimental work for determining the Young Modulus.
\end{abstract}

Index Terms-Haptic interfaces, virtual experiment, virtual reality, Young Modulus.

\section{INTRODUCTION}

In the context of undergraduate engineering courses, it is difficult for many students to establish the relation between stiffness and Young Modulus and to compare them for different materials. However, in the area of Materials Engineering and Technological Processes, the Young Modulus is a relevant property $[1,2]$. The HapticBender system has come out as a new teaching and learning tool, allowing students to have practice and to determine the Young Modulus of materials. The novel feature of such tool is the possibility of offering different materials and thereby establishing a way for students to "feel" and contrast different materials based on haptic feedback.

The HapticBender uses virtual reality and haptics technology [2] and is an evolution of the system presented in [2]. In the actual version the set-up can be rotated and viewed from different angles, increasing the user perception about the real system and potentiating the user interaction with the experiment.

Virtual reality allows the simulation of something real, permitting, at the same time, the study of its dynamical behavior. In certain cases, a virtual reality application might even be richer than its real counterpart, thanks to new features that are possible only in the context of virtual, mixed and augmented reality.

Haptic devices, on the other hand, can potentiate the realism of the virtual applications and offer interaction outputs similar to those achieved in real experiments. Haptic devices have been used in education and training activities that depend on touch, to get a better understanding of the involved phenomena [2-6].

\section{THE HAPTICBENDER}

The HapticBender is a virtual replica of a real experiment. It comprises a cantilever beam under bending test. The user can apply different loads at the free end of the beam, through a haptic device and feel realistic reaction forces. In the user graphical interface the values of the applied load and strain are displayed and, using the beam geometry parameters, the Young Modulus can be calculated. Two materials (aluminum and nylon) can be tested. These materials have been selected because the first is a common material in engineering design and production and Nylon presents a Young Modulus near 40 times lower than the Aluminum, convenient for asset the property contrast.

In the present version the HapticBender allows the rotation of the set-up comprising the beam and its support. This new feature allows the user to observe beam deformation from different viewpoints and have a complete perception regarding the beam dynamic (Fig. 1). Moreover, with this feature, associated to the richer graphical interface and force feedback (Fig. 2), the user will get a deeper immersion in the experiment, taking advantage of all available information to learn, consolidate and get motivation to evaluate the Young Modulus of materials and correlate values from different materials with the different beam reaction forces.

The effect of rotation was implemented by rotating the view (camera) around the virtual object. This procedure has the advantage of ensuring that both the material and the color properties are not lost. In other words, during the rotation, the coordinates of the object are modified but the data corresponding to the design properties (materials and colors) are kept unchanged.

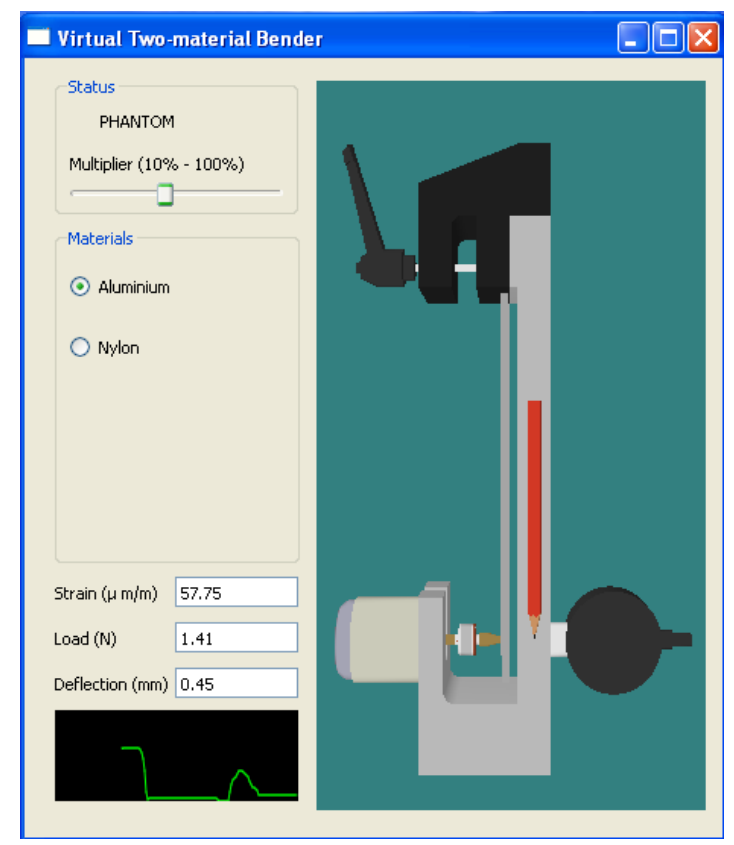

Figure 1. HapticBender system. 


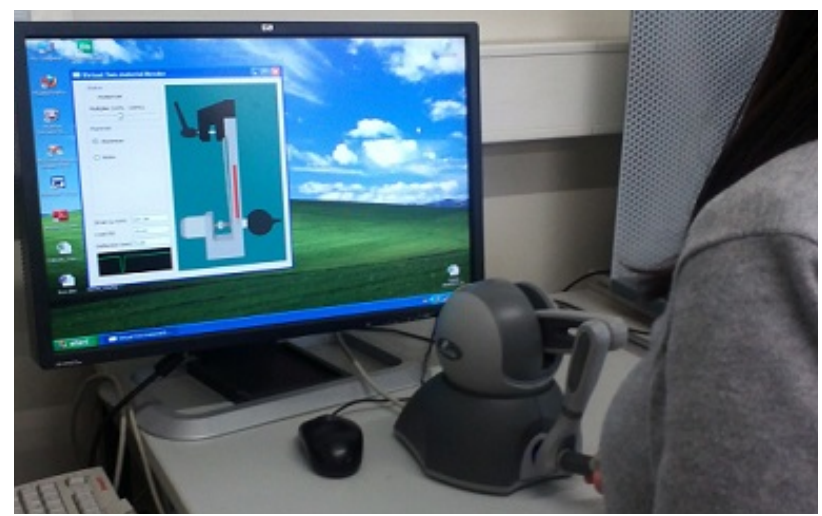

Figure 2. Interacting with the HapticBender system.

The virtual object was created using the SolidWorks CAD software, generating a file with all information about the object design. The HapticBender just interprets that information reading the file. In the sequel, the system moves the object treating all design properties as static data. When applying the rotation to the view, the object coordinates are not affected and the rotation is possible.

The HapticBender allows the user to easily visualize and manipulate the virtual object using the mouse. Mouse movements are interpreted by the application as events. This means that pressing the right button over the virtual object and moving the mouse to the right will turn the camera to the right around the object vertical axis. Conversely, pressing the left button and moving the mouse to the left will turn the camera to the left (Fig. 3).

\section{IMPACT OF USE ON LEARNING}

In order to assess the effects of using the described setup during the learning processes of Young Modulus, a small, knowledge-based questionnaire was used. This included a 6 item multiple-choice section, as well as 2 Likert scales to evaluate the perceived rigidity and intensity needed to deform materials. The students' perceptions and opinions about the setup itself were also assessed with the use of Likert scales and used as complementary measures of subjective satisfaction.

The experimental design included the use of three distinct groups: the Control group, exposed only to the theoretical contents, the "Haptic" group, which executed the virtual experiment with the HapticBender and the "Remote" group, which used a remote set-up, virtually replicated by HapticBender, without any Haptic interaction. Given the specificity of the current paper, only the results from the latter groups will be discussed.

\section{RESUlts}

The sample of participants included 95 students (34 in the Control group, 30 in the "Haptic" and 31 in the "Remote" groups). The students were randomly recruited in a second year discipline from mechanical engineering course in the Faculty of Engineering of University of Porto, who were assigned to the groups after a theoretical class were the determination method procedure were explained. The mean age was 18 years $(\mathrm{SD}=0.709)$ and $89.5 \%$ participants were male.

Concerning the knowledge-based assessment, the Haptic group $(\mathrm{M}=4.67, \mathrm{SD}=0.994)$ had a significantly higher number of correct answers $(\mathrm{p}=0.037)$ than the Remote group $(\mathrm{M}=3.94, \mathrm{SD}=1.181)$.

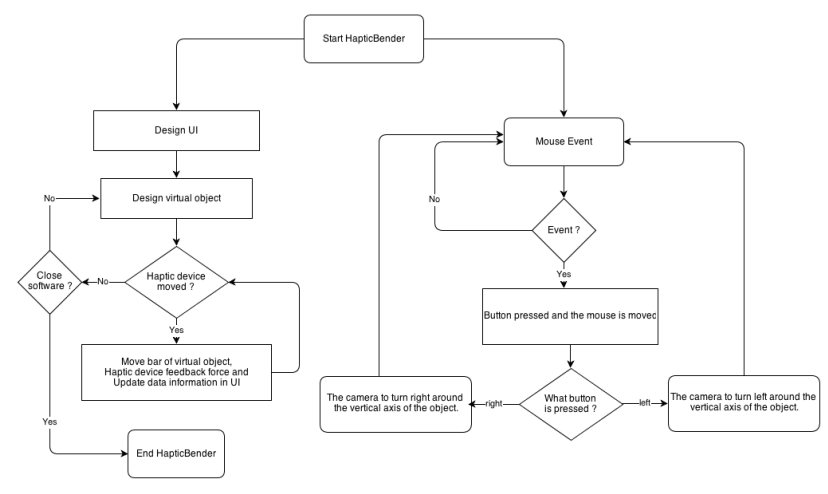

Figure 3. Schematic representation of the implemented software.

In the perception of rigidity (coded as the difference between the perceived rigidity of Aluminium and Nylon beams $)$, the Haptic group $(\mathrm{M}=4.57, \mathrm{SD}=2.128)$ presented significantly higher values $(p=0.001)$ than the Remote group. The same result was found when comparing the perception of intensity needed to deform the material (coded as the difference between perceived intensity of Aluminium and Nylon) - the Haptic group $(\mathrm{M}=4.20, \mathrm{SD}$ $=2.455)$ presented higher values $(\mathrm{p}=0.002)$ than the Remote group $(\mathrm{M}=0.8, \mathrm{SD}=3.882)$.

The evaluation of students' opinion about the experience and the perceptions about it points that the Haptic group presented higher values when assessing the contribution of this kind of systems as an aid to understand differences between materials $(p<0.001)$ and the force needed to deform each of them $(p=0.001)$. At the same time, the Haptic group also perceived the experience as being more useful to understand the materials $(p=0.008)$, their differences $(p<0.001)$ and underlined the relevance of participating in similar experiences $(p=0.01)-$ when compared with the assessment done by the Remote group.

All the results reflect the $\mathrm{T}$ test for independent samples, significance values.

\section{CONClusion}

The HapticBender allows the user to interact with a virtual cantilever beam, applying forces and feeling the beam reaction, using a haptic device. In the user graphical interface the application provides all data necessary to compute the Young Modulus, namely the load and strain, and to predict the deflection that will occur on the beam. Two materials can be tested: aluminum and nylon. A benefit of the virtual tool is that it can replace a real one, allowing portability, since it can be downloaded and installed in any computer with a haptic device (presently, it is used a PHANTOM Omni or PHANTOM Premium). It also can be accede remotely in a server.

The main results from this experience seem very clear: the group exposed to the HapticBender system presented consistently higher results in almost all the used measures. The sensorimotor feedback seems to have had a positive impact both in the knowledge acquisition as well as in the perception of the experience itself.

The results go very much in line with the general literature regarding the effects of practice in knowledge acquisition [8-9]. The higher interaction and feedback allows for a better memorization of the concepts than the pure visual setting - this has been suggested as a possible result of multi-sensorial information integration, and some stud- 
ies have already found similar increased knowledge learning with the use of haptic devices [10].

Although the experimental design could be improved and a deeper assessment strategy could be designed, these results strongly point towards the positive effect of using Haptic systems to increase learning performance and further testing scenarios should be pursued.

\section{ACKNOWLEDGMENT}

The present work is due to the development and innovation activities in online experimentation within the project Experiment@Portugal2102 funded by Calouste Gulbenkian Foundation and in agreement with other different activities within PEst-OE/EME/LA0022/2013, FCT.

\section{REFERENCES}

[1] J. N. Ding, Y. G. Meng, S. Z. Wen, "Experimental and theoretical study of Young modulus in micromachined polysilicon films", Tsinghua Science and Technology, vol. 7, pp. 270-275, 2002.

[2] M. T. Restivo, A. M. Lopes and P. J. Xia, “"Feeling” Young Modulus of Materials", Rev2012 Conference, Bilbao, Spain, pp 412-415, 2012.

[3] Ravikiran B. Singapogu and Timothy C. Burg, "Haptic Virtual Manipulatives for Enhancing K-12 Special Education", in Proceedings of the 47th Annual Southeast Regional Conference ACM-SE 47, New York, USA, 2009.

[4] R.L. Williams, X. He, T. Franklin, and S. Wang, "HapticsAugmented Engineering Mechanics Educational Tools," World Transactions on Engineering and Technology Education, vol.6, 2007.

[5] O. Goldstain, I. Ben-Gal and Y. Bukchin, "Remote learning for the manipulation and control of robotic cells," European Journal of Engineering Education, vol. 32, n. 4, pp. 481-494, August 2007. http://dx.doi.org/10.1080/03043790701337213

[6] C. A. Jara, F. A. Candelas, S. T. Puente and F. Torres, "Hands-on experience of undergraduate students in automatics and robotics using a virtual and remote laboratory," Computers \& Education
57, pp. 2451-2461, 2011. http://dx.doi.org/10.1016/j.compedu. $\underline{2011.07 .003}$

[7] M.T. Restivo, J. Mendes, A.M. Lopes, C.M. Silva, and F. Chouzal, "A Remote Lab in Engineering Measurement," IEEE Trans. on Industrial Electronics, vol. 56, pp 4436-4843, 2009. http://dx.doi.org/10.1109/TIE.2008.2011479

[8] Glover, J., Ronning, R., Bruning, R, "Cognitive Psychology for Teachers", 1990

[9] Kalchman, M., Moss, J., Case, R. "Psychological models for the development of understading: rational numbers and functions", Cognition and Instruction: Twenty-five years of progress, pp 1-38

[10] Hamza-Lup, F.G., "Feel the pressure: e-learning systems with haptic feedback", Haptic interfaces for virtual environment and teleoperator systems, pp 445-450, 2008.

\section{AUTHORS}

Maria Teresa Restivo, $\mathrm{PhD}$ in Engineering Sciences, is with Faculty of Engineering, University of Porto, Portugal and IDMEC - Pólo FEUP (trestivo@fe.up.pt).

António M. Lopes, PhD in Mechanical Engineering, University of Porto, Portugal, is with Faculty of Engineering, University of Porto and IDMEC - Pólo FEUP (aml@fe.up.pt).

Linda Padilha, Integrated Master Student of Informatics and Computer Engineering of Faculty of Engineering, University of Porto, Portugal (linda.anthu@gmail.com).

Pedro Chaves, PhD student at Faculty of Psychology and Education Sciences, University of Porto, Porto, Portugal (pedromlchaves@gmail.com).

Teresa Duarte, $\mathrm{PhD}$ in Mechanical Engineering, University of Porto, Portugal, is with Faculty of Engineering, University of Porto, Portugal and Institute of Mechanical Engineering and Industrial Management (tpd@fe.up.pt)

This article is an extended and modified version of a paper presented at the International Conference exp.at'13, held 18-20 September 2013, in Coimbra, Portugal. Submitted 18 November 2013. Published as resubmitted by the authors 04 December 2013. 